\title{
Serologic Investigation of Pneumococcal Immunization of Autologous Hematopoietic Stem Cell Recipients
}

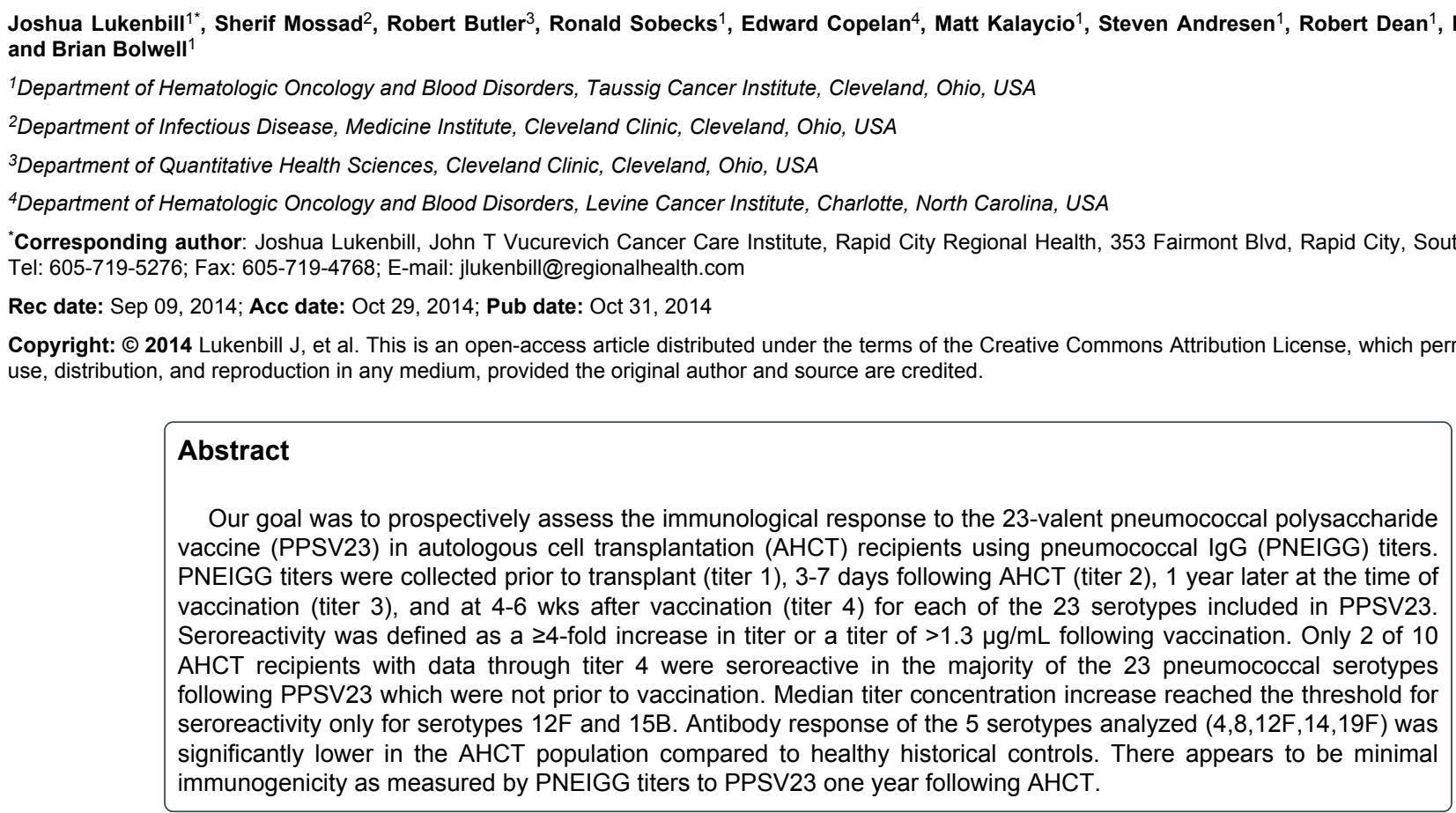

\section{Introduction}

Autologous hematopoietic cell transplantation (AHCT) recipients are vulnerable to many infections, including Streptococcus pneumonia, given the inherent immunosuppression associated with this procedure. Kumar et al. analyzed 1238 patients following HCT, of whom 9 of $431(2.1 \%)$ allogeneic and 5 of $807(0.06 \%)$ autologous recipients developed invasive pneumococcal disease [1]. Therefore, primary prevention with vaccination is paramount.

This prospective study's goal was to provide further insight into the immune response to the 23-valent polysaccharide vaccine (PPSV23, Pneumovax 23, Merck \& Co., Whitehouse Station, NJ) in AHCT recipients. This was assessed by measurement of seroreactivity following vaccination, as quantified by pneumococcal IgG (PNEIGG) antibody titers. Of note, this study was designed and conducted to allow for PPSV23 at 1 year post-HSCT, as per the 2000 Centers for Disease Control and Prevention/Infectious Disease Society of America/American Society for Blood and Marrow Transplantation recommendations [2]. The primary outcome was PNEIGG at 4-6 weeks after PPSV23. We also collected PNEIGG titers at 3 other time points: before AHCT, following stem cell infusion, and 1 year later at the time of vaccination. Finally, we compared our AHCT population's PNEIGG response following vaccination to that of historical healthy controls.

\section{Materials and Methods}

Fifty patients with lymphoma and multiple myeloma undergoing AHCT at the Cleveland Clinic between June 2009 and March 2010 were recruited. Patients were treated on AHCT protocols that had previously been approved by the Cleveland Clinic's Institutional Review Board with signed informed consent obtained from all patients. In addition, written informed consent for participation in this study was obtained from all subjects. Historic control data regarding the immunologic response to PPSV23 in healthy elderly persons was extracted from a study by Carson et al. [3]. This study was designed to analyze the differential immunological response of young and healthy persons to PPSV23. PNEIGG data were collected pre-vaccination and then 4 weeks after vaccination for five serotypes $(4,8,12 \mathrm{~F}, 14,19 \mathrm{~F})$. The PNEIGG results from both arms in that study were combined for the 50 controls then compared to out AHCT study population for those 5 serotypes.

Titer 1 was collected during pre-transplant evaluation, prior to hospitalization for AHCT. Titer 2 was collected 3-7 days following stem cell infusion. Titer 3 was collected at the time of pneumococcal vaccination 1 year following AHCT. Finally, Titer 4 was collected 4-6 weeks after vaccination. These samples were then allowed to coagulate at room temperature for 30 minutes, followed by a 10 minute $1300 \mathrm{~g}$ centrifuge run at $4^{\circ} \mathrm{C}$. The serum supernatant was then aliquoted into two $1.8 \mathrm{~mL}$ cryovials then immediately frozen at $-80^{\circ} \mathrm{C}$. These samples were sent for PNEIGG measurement (Mayo Medical Laboratories, Rochester, MN). At 1 year post-AHCT, participants were offered PPSV23. There were no unexpected complications of vaccination in any of the subjects.

The sample size calculation was based on data from Mufson et al. on pneumococcal antibody titers following vaccination [4]. In order to achieve a power of $80 \%$ and an alpha of .05 , a sample size of 42 was required (mean of the differences $=1.1$, population standard deviation=2.7); therefore, our goal accrual was 50 subjects. For each of 
the 23 serotypes analyzed, seroreactivity was defined as a titer $>1.3$ $\mu \mathrm{g} / \mathrm{mL}$, or a fourfold increase in titer 3 (pre-vaccination) to titer 4 (post-vaccination) [5]. Two way ANOVA methods using subjects and titers to adjust for repeated measures within a subject were used to assess differences in titer results. Contrasts were constructed to test specific pair-wise means (baseline vs. initial, baseline vs. 1 year later, 1 year later vs. 1.1 years later, and post-vaccination). One sample equivalence-test was used to compare antibody titers of AHCT recipients 4-6 weeks post-vaccination to historical healthy controls. Assessment of differences between the data by Carson et al. [3] and our study's titers 3 and 4 were run using one sample t-tests with derived target null values. Results were not adjusted for multiple comparisons. Differences with $\mathrm{p}<.05$ were considered to be statistically significant.

\section{Results}

Fifty patients were enrolled to participate in the study. However, 40 patients dropped out prior to completion of the study, most commonly due to continuation of care at a community oncology clinic (24 patients). Of the remaining 10 patients, the median age was 56, 9 were male, 6 had multiple myeloma, and 4 had non-Hodgkin lymphoma. The median number of previous chemotherapy regimens was six, one had prior AHCT, one had previous radiotherapy, one with previous pneumococcal vaccination, and one had previously documented pneumonia.

Only two patients acquired seroreactivity to a majority of serotypes in response to vaccination (comparison of Titer 3 to 4 ). Four patients maintained the significant seroreactivity that they had prior to vaccination. Finally, four patients had minimal seroreactivity, both prior and following vaccination. Analysis of the median change in titer concentrations revealed minimal variation at the three intervals as well. Only serotypes $12 \mathrm{~F}$ and $15 \mathrm{~B}$ had a median titer increase from titer 3 to titer 4 which reached the threshold for seroreactivity. Finally, our data were compared to the healthy historical controls in the study by Carson et al. [3], revealing significantly different mean serotype responses to PPSV23 to all 5 compared serotypes ( $\mathrm{p}<.0001$ ), as shown in the Table 1 . All five serotypes $(4,8,12 \mathrm{~F}, 14,19 \mathrm{~F})$ in the control data achieved the definition of seroreactivity, but only serotype $12 \mathrm{~F}$ (as well as 15B) were seroreactive in our study population.

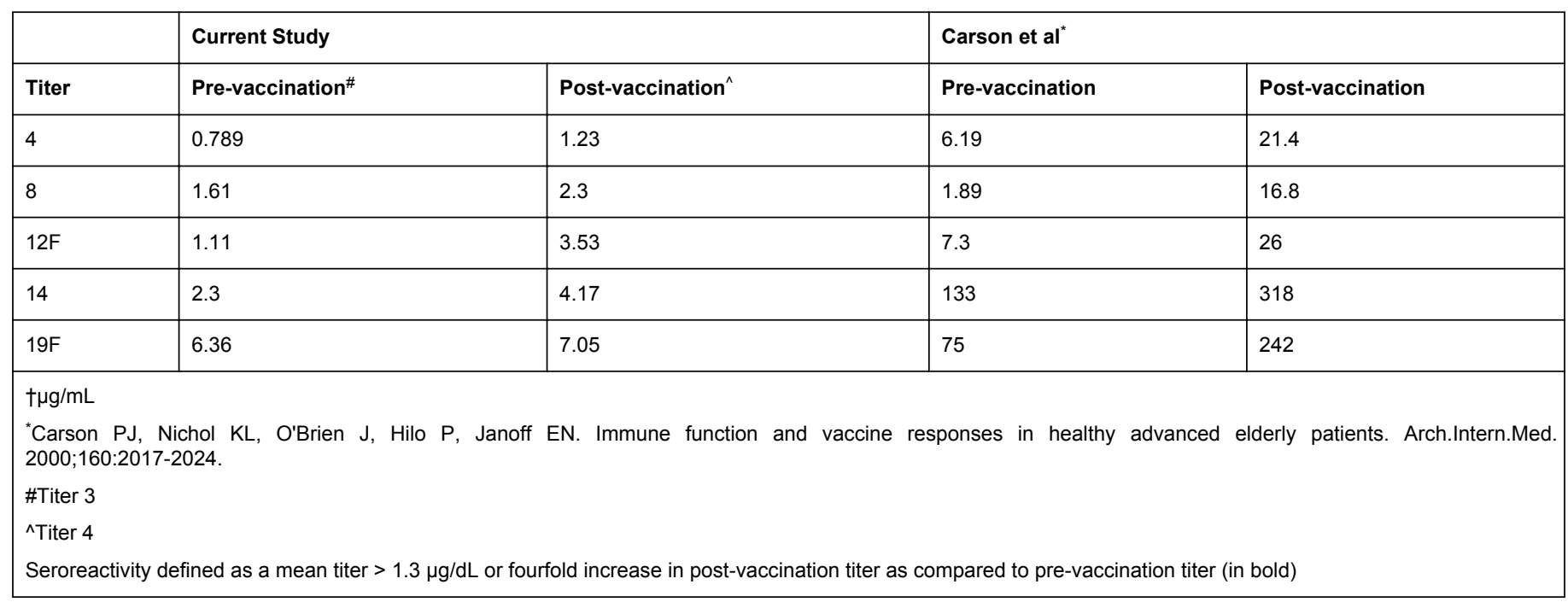

Table 1: Comparison of mean titer concentrations $\dagger$ of AHCT recipients to healthy controls ${ }^{*}$

\section{Discussion}

This study revealed minimal immunogenicity to PPSV23, as measured by number of subjects with seroreactivity in the majority of titers, the number of serotypes with seroreactivity across all subjects, and in comparison with historic controls. The primary limitation of this study is that it failed to reach the goal sample size of 42 patients. Patients' dropout was attributable to may causes, including progressive disease, co-morbidities, and death; however, the primary reason was lack of patient follow-up. This lack of data for Titers \#3 and 4 compromises the external validity of our results.

The AHCT population has not been well-studied; however, this trend of poor immunogenicity to PPSV23 appears to extend to all of the hematopoietic stem cell transplant population. A study by Kumar et al. including autologous and allogeneic transplant patients, found that the serotypic response to PPSV23 was inferior to the conjugate vaccine PCV7 (Prevnar, Wyeth Pharmaceuticals Inc.) Philadelphia,
PA) at 12 months post-transplant $(\mathrm{p}=.003)$ [6]. The Advisory Committee on Immunization Practices recently endorsed PCV13 (PCV13, Prevnar 13, Wyeth Pharmaceuticals Inc., Philadelphia, PA) as the preferred vaccine in adults $\geq 19$ [7]. However, administration of PPSV23 after the series of 3 doses of PCV13 has been shown not only to increase serotype coverage but also to increase the immunogenicity to serotypes included in the conjugate vaccine [8]. Therefore, the current recommendation for all hematopoietic cell transplant recipients is to receive a series of 3 administrations of the PCV13 vaccine, followed by the PPSV23 vaccine 8 weeks later $[7,9]$.

The primary goal of this study was to assess the immunologic response to PPSV23 in patients undergoing AHCT. Though our sample size was limited, our data support that PPSV23 vaccination alone induces minimal immunogenicity as measured by PNEIGG. It is our hope that this study and others on the topic will increase our knowledge of the efficacy of pneumococcal vaccination in AHCT recipients. 
Citation: Lukenbill J, Mossad S, Butler R, Sobecks R, Copelan E, et al. (2014) Serologic Investigation of Pneumococcal Immunization of Autologous Hematopoietic Stem Cell Recipients . Clin Microbiol 3: 177. doi:10.4172/2327-5073.1000177

Page 3 of 3

\section{Acknowledgement}

Supportive foundations are National Institutes of Health, National Center for Research Resources, CTSA 1UL1RR024989, Cleveland, Ohio.

\section{References}

1. Kumar D, Chen MH, Welsh B, Siegal D, Cobos I, et al. (2007) A randomized, double-blind trial of pneumococcal vaccination in adult allogeneic stem cell transplant donors and recipients. Clin Infect Dis 45: 1576-1582.

2. Centers for Disease Control and Prevention, Infectious Diseases Society of America, and American Society of Blood and Marrow Transplantation (2000). Guidelines for preventing opportunistic infections among hematopoietic stem cell transplant recipients. Biol. Blood Marrow Transplant, G: 659-713; 715; 717-727.

3. Carson PJ, Nichol KL, O'Brien J, Hilo P, Janoff EN (2000) Immune function and vaccine responses in healthy advanced elderly patients. Arch Intern Med 160: 2017-2024.

4. Mufson MA, Krause HE, Schiffman G, Hughey DF (1987) Pneumococcal antibody levels one decade after immunization of healthy adults. Am J Med Sci 293: 279-284.

5. Sorensen RU, Leiva LE, Javier FC, 3rd, Sacerdote DM, Bradford N, Butler B, Giangrosso PA, and Moore C (1998).Influence of age on the response to Streptococcus pneumoniae vaccine in patients with recurrent infections and normal immunoglobulin concentrations. J.Allergy Clin.Immunol, 102: 215-221.

6. Kumar D, Humar A, Plevneshi A, Siegal D, Franke N, et al. (2008) Invasive pneumococcal disease in adult hematopoietic stem cell transplant recipients: a decade of prospective population-based surveillance. Bone Marrow Transplant 41: 743-747.

7. Centers for Disease Control and Prevention (CDC) (2012) Use of 13valent pneumococcal conjugate vaccine and 23 -valent pneumococcal polysaccharide vaccine for adults with immunocompromising conditions: recommendations of the Advisory Committee on Immunization Practices (ACIP). MMWR Morb Mortal Wkly Rep 61: 816-819.

8. Cordonnier C, Labopin M, Chesnel V, Ribaud P, Camara Rde L, et al. (2010) Immune response to the 23 -valent polysaccharide pneumococcal vaccine after the 7-valent conjugate vaccine in allogeneic stem cell transplant recipients: results from the EBMT IDWP01 trial. Vaccine 28: 2730-2734.

9. Centers for Disease Control and Prevention (CDC); Advisory Committee on Immunization Practices (2010) Updated recommendations for prevention of invasive pneumococcal disease among adults using the 23 valent pneumococcal polysaccharide vaccine (PPSV23). MMWR Morb Mortal Wkly Rep 59: 1102-1106. 